\title{
Stroboscopic and Voice Analysis for Outcome Assessment of Intervention in Cases with Benign Vocal Fold Lesions
}

\author{
Neha Raghuwanshi ${ }^{1}$, Aparaajita Mundra ${ }^{2}$, Neeraj K Dubey ${ }^{3}$, Surbhi Godha ${ }^{4}$, Rajkumar Mundra ${ }^{5}$
}

\begin{abstract}
Aims and objectives: Assessment of outcome of cases with benign vocal lesions treated by a combination of methods aimed at voice improvement, rather than only cure of lesion.

Materials and methods: A prospective study was conducted in the Department of Otorhinolaryngology, MGM Medical College and Maharana Yashwant Rao Holkar Hospital, Indore from October 2018 to March 2020. We selected 85 patients in the age group of 18-60 years with complaints of hoarseness of voice and in whom benign vocal fold lesions were observed on stroboscopy. Pretreatment analysis was done by video-stroboscopy and voice analysis by a combination of simple subjective and objective parameters. Treatment was planned individually for each case from a variety of available modalities, with vocal fold exercises being the essential part of the plan in each case. After 2 months from the initiation of therapy, the analysis was repeated and compared with initial observations. The data were recorded and compared using paired $t$-test.

Results: Statistically significant $(p<0.05)$ improvement was observed in all the parameters of stroboscopic and voice analysis.

Conclusion: The contemporary approach of benign vocal fold lesions is aimed at cure of lesion with the achievement of the best possible voice outcome. This makes voice therapy an essentially irreplaceable part of the treatment plan.

Clinical significance: Establishment of the importance of voice therapy and patient cooperation in the treatment of hoarseness.

Keywords: Benign vocal fold lesions, Maximum phonation time, Microlaryngeal surgery, s/z Score, Vocal function exercises.

International Journal of Phonosurgery \& Laryngology (2021): 10.5005/jp-journals-10023-1195
\end{abstract}

\section{INTRODUCTION}

The normal phonatory phenomenon requires proper structure, physiology, coordination, and efficiency of the larynx. A complication in any of these properties has adverse effects on phonation.

The term dysphonia is often used interchangeably with hoarseness. However, this terminology is not precise, as hoarseness is a symptom of a change in voice quality reported by patients, while dysphonia indicates impairment of voice quality as recognized by a clinician. ${ }^{1}$

The most widely accepted and predominant cause of hoarseness is benign lesions of vocal folds, which affect vibratory vocal fold function and result in significant dysphonia. ${ }^{2}$ The treatment for such lesions includes a combination of microlaryngeal surgery, voice therapy, low dose steroid therapy, and lifestyle modifications like quitting smoking and elimination of behavior aggravating phonotrauma. Contributing factors like allergy, reflux, and psychological issues need to be addressed. ${ }^{3}$

Voice therapy has acquired an essential role in the treatment of dysphonia. Holistic voice therapy is based on a continuum of voice wellness from a disordered to an elite voice. Such therapy attends to all the three sub-systems of voice production-respiration, phonation, and resonance. Vocal function exercises (VFE) is such holistic therapy that is effective in improving disordered voice and enhancing normal voice. ${ }^{4}$ These exercises are believed to balance and re-strengthen laryngeal musculature, improve flexibility and movements of vocal folds and rebalance the airflow. ${ }^{5}$

A detailed evaluation includes visualization of the larynx and voice assessment. Stroboscopy provides visualization of vocal folds, mucosa, closure pattern, and lesions. ${ }^{6}$

\footnotetext{
${ }^{1-5}$ Department of ENT, MGM Medical College, Indore, Madhya Pradesh, India
}

Corresponding Author: Aparaajita Mundra, Department of ENT, MGM Medical College, Indore, Madhya Pradesh, India, Phone: +91 9669110309, e-mail: draparaajita6@gmail.com

How to cite this article: Raghuwanshi N, Mundra A, Dubey NK, et al. Stroboscopic and Voice Analysis for Outcome Assessment of Intervention in Cases with Benign Vocal Fold Lesions. Int J Phonosurg Laryngol 2021;11(1):16-20.

Source of support: Nil

Conflict of interest: None

Voice assessment is an important part of the examination for laryngeal pathology. Various methods of both subjective and objective analysis are used for voice analysis. The simplest of all objective methods are the maximum phonation time (MPT) and s/z score.

Maximum phonation time is measured by asking the subject to inhale as deeply as possible and then sustain a steady $/ \alpha /$ vowel (as in far) for as long as possible. The longest of three repeated measurements is selected. Values under 10 seconds are regarded as pathological. It is presumed to provide a rough indication of respiratory support and phonatory function. It is a non-invasive and quick test. ${ }^{7,8}$

$\mathrm{S} / \mathrm{z}$ score is the ratio of maximum sustained time for a speaker to make both an /s/ and /z/ sound. A normal speaker can usually maintain the sound for approximately the same amount of time for both /s/ and voiced / $z$ / consonant, giving a ratio of approximately 1:1. Abnormalities that interfere with vocal fold vibration or which affect 
glottic closure reduce the $/ z$ / value but do not significantly affect the $/ \mathrm{s} /$ time, thus increasing the ratio. This simple test is used quite widely in clinical practice. ${ }^{9}$ The dysphonic subjects with laryngeal pathology usually have $s / z$ ratios above $1.4 .^{10}$

The VRQO-L questionnaires consist of 10 statements on voicerelated characteristics across emotional, physical, and functional domains. Patients respond according to the suitability or closeness of each point (ranging from $1=$ not a problem to $5=$ the problem is "as bad as it can be") to their situations. Thus, the VRQO-L score ranges from 10 to 15 (excellent), 16 to 20 (very good), 21 to 25 (good), 26 to 30 (fair), and scores $>30$ and up to 50 is poor. ${ }^{11}$

In this study, we aimed to analyze the results of our interventions, which were a combination of treatment modalities, in cases with benign vocal fold lesions.

\section{Aims and Objectives}

- Use of video-stroboscopy for early detection and identification of benign vocal fold lesions.

- Analysis of voice for understanding the severity of voice disorders.

- Follow-up and assessment of results in terms of videostroboscopic and voice assessment parameters.

\section{Materials and Methods}

This study was a prospective study conducted in the Department of Otorhinolaryngology, MGM Medical College and Maharana Yashwant Rao Holkar Hospital, Indore from October 2018 to March 2020.

We selected 85 patients in the age group of 18-60 years with complaints of hoarseness of voice and in whom benign vocal fold lesions were observed on indirect laryngoscopy or videostroboscopy.

\section{Exclusion Criterion}

- Known cases of TB.

- History of any laryngeal trauma.

- Laryngeal conditions like acute infections, malignancies, or vocal cord palsy.

- Disorders of articulation.

- Uncooperative patients, patients with an excessive gag reflex, or those not giving consent.

- Patients with any systemic comorbidities.

Patients with voice complaints were examined by indirect laryngoscopy, video-laryngoscopy, and video-stroboscopy, and voice assessment by MPT and $s / z$ score and response to VRQOL questionnaire. After proper evaluation, the cases underwent appropriate treatment which included a combination of the following: microlaryngeal surgery, voice therapy including VFE, low dose steroid therapy, proton pump inhibitors, and lifestyle modifications like quitting smoking, vocal hygiene. Cold-knife surgery microlaryngeal and excision of vocal fold lesions was performed in 26 cases, essentially including all cases with polyps and leukoplakia. After surgery, the excised specimen was sent for histopathological analysis. Every case was taught and encouraged to do VFE, even following cure of lesion.

Post-therapy analysis was done after 2 months of treatment using video-stroboscopy, voice analysis by MPT and s/z score, and a second response to the VRQOL questionnaire was noted.
Data were compiled in an excel sheet and paired $t$-test was used to find the statistical significance of the observations. The $p$ value $<0.05$ is considered to be statistically significant.

\section{Vocal Function Exercises: In Brief}

Based on Stemple's principles of a holistic approach to voice therapy: ${ }^{4}$

These are a series of four exercises to be practiced at home, two times each, twice per day, preferably morning and evening. These exercises include:

- Warm-up exercise-Sustain the /i/ vowel for as long as possible on a musical note $(F)$ above the middle $(C)$ for females and boys, $(F)$ below the middle (C) for adult males. (Notes may be modified up or down to fit the needs of the client. Seldom are they modified by more than two notes in either direction.)

Goal: Based on airflow volume. When airflow measures are not available, the goal is equal to the longest $/ s /$ that the case can sustain.

- Stretching exercise-Glide from the lowest note to the highest possible note on the word "knoll".Goal: No voice breaks.

- Contracting exercise-Glide from the highest note to the lowest note on the word "knoll".Goal: No voice breaks.

- Low-Impact Adductory Power Exercise-Sustain the musical notes (C-D-E-F-G) for as long as possible on the word "knoll" minus the "kn". (Middle C for females and boys, an octave below middle $C$ for males.)

Goal: Remains the same as for exercise number 1 .

The tone quality was monitored for voice breaks and breathiness. All exercises were to be performed as softly as possible.

Also, cases were asked to plot their weekly progress on the graphs provided. The program usually lasts for $6-8$ weeks.

When the patient reached the predetermined therapy goal, and voice quality improvement was observed, then a tapering maintenance program was recommended.

\section{Results}

Out of the total 85 cases included in our study, 53 were males and 32 were females, with a male to female ratio of 1.65 . Most cases belonged to the age group of 31-40 (31.5\%) years.

A positive history of vocal abuse was found in 62 (72.9\%) cases and 42 cases (49.4\%) were smokers. Symptoms of LPR (dryness in throat, heartburn, dry cough, foreign body sensation in throat) were found in 53 cases (62.3\%).

A variety of vocal fold lesions were observed on stroboscopy, vocal nodules ( $n=42$ ) being the most common ones, followed by polyps ( $n=18)$, as shown in Figure 1. Other vocal fold lesions observed are vocal fold leukoplakia $(n=9)$, cyst $(n=8)$, and sulci $(n=7)$. The least commonly observed lesions were vocal fold erythema $(n=2)$, Reinke's edema $(n=2)$, and papilloma $(n=2)$. Figures 2 to 5 show images of lesions observed on stroboscopy. Figure 2 shows open and closed phases, respectively, of a case of a broad-based polyp on right vocal fold. Figure 3 shows the left vocal fold cyst. Figure 4 shows the right vocal fold nodule with approximating false vocal folds. Figures 5 and 6 show intraoperative pictures of left vocal fold papilloma and left vocal fold polyp, respectively.

In the present study, the most common site of lesion was anterior one-third with 54 cases. In 15 cases, diffuse involvement was observed, whereas the middle third in 13 cases. The least affected site was posterior third, only in three cases. 


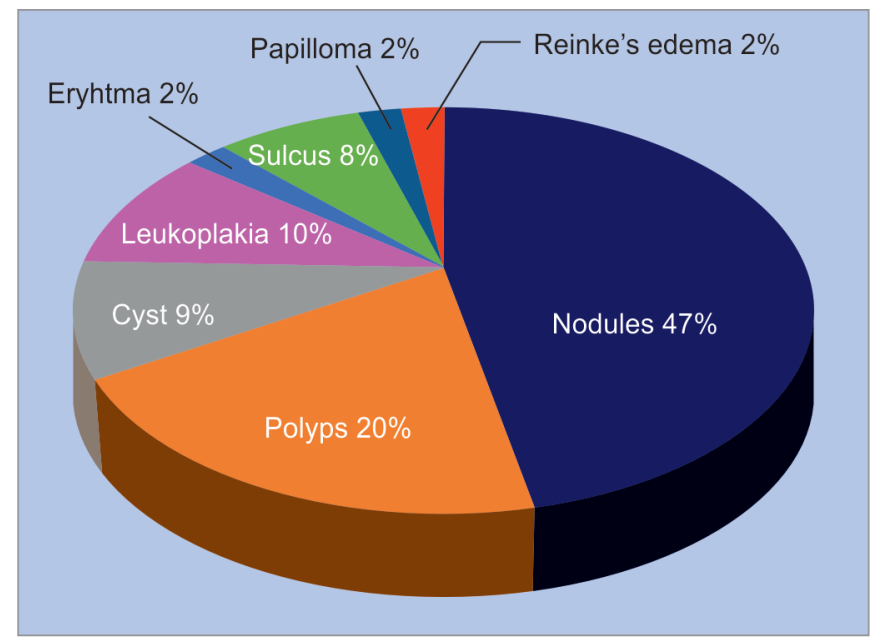

Fig. 1: Distribution of various lesions

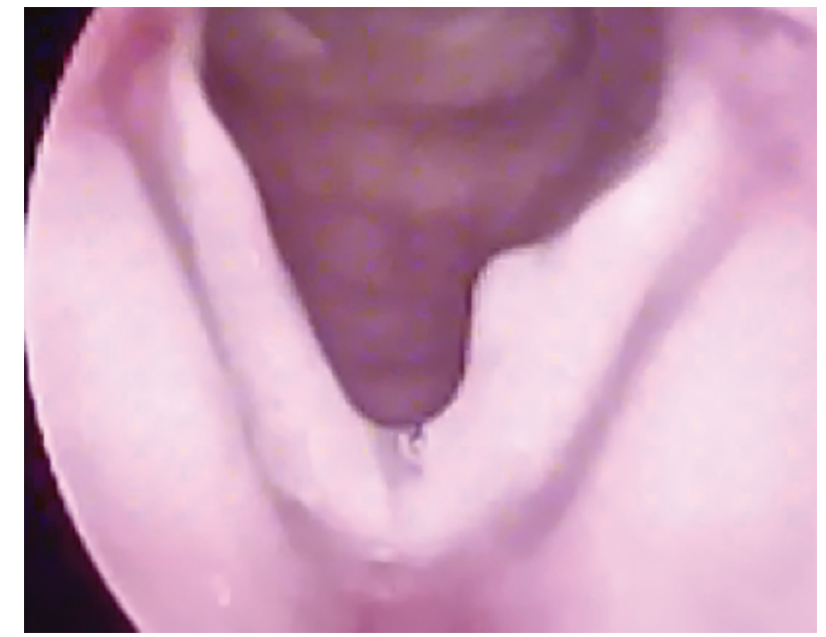

Fig. 3: Left vocal fold cyst

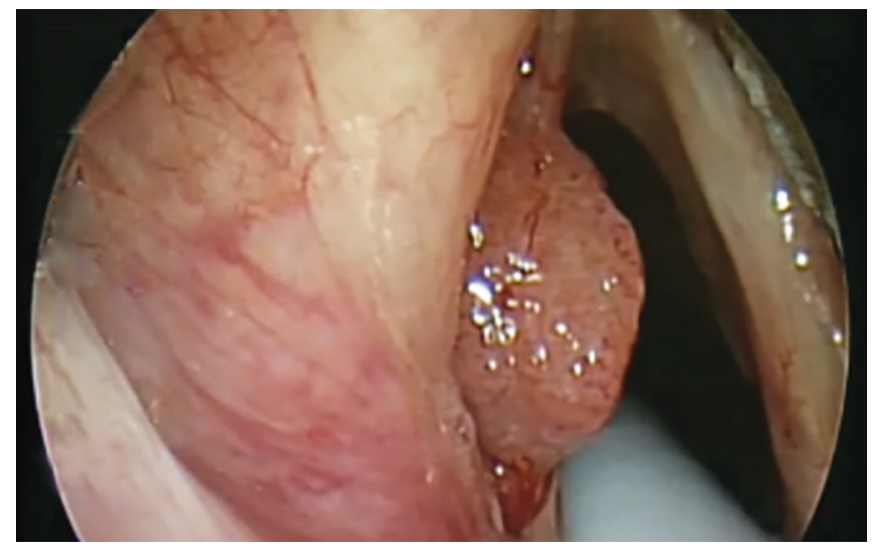

Fig. 5: Intraoperative picture of left vocal fold papilloma

\section{Voice Analysis}

MPT was enhanced from the mean of 8.2 seconds to a mean of 17.5 seconds after treatment. The $t$ value is 34.65 and the $p$ value is 0.00001 , which is statistically significant.

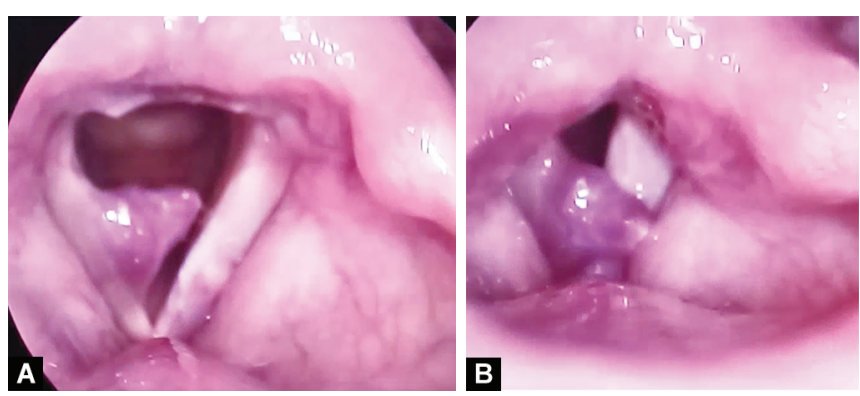

Figs $2 \mathrm{~A}$ and B: Broad based right vocal fold polyp: (A) Open phase (B) Closed phase

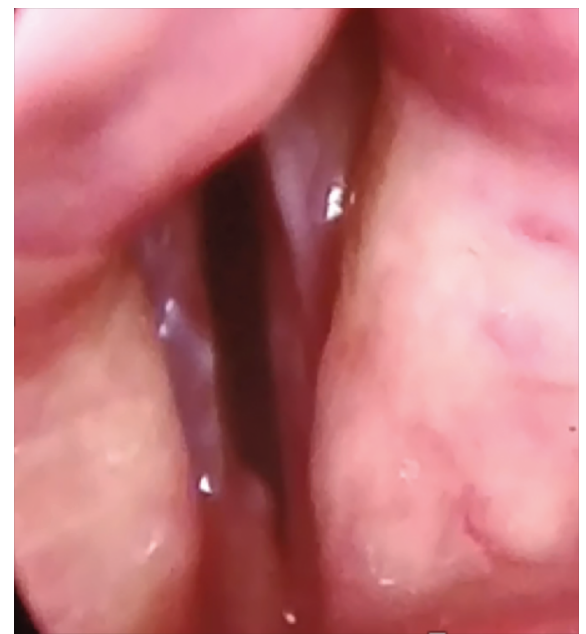

Fig. 4: Right vocal fold nodule with approximating false vocal folds

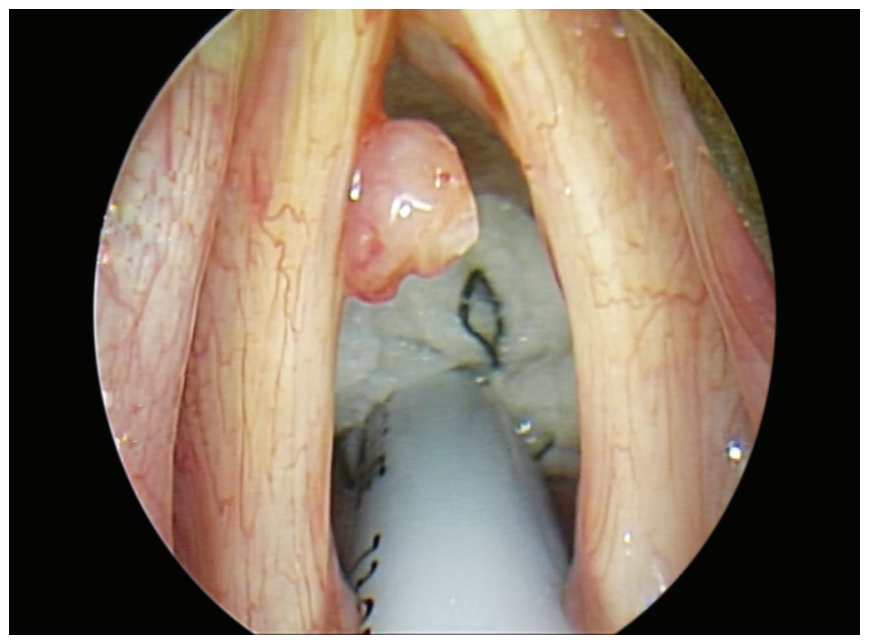

Fig. 6: Intraoperative picture of left vocal fold polyp

Similarly, improvement in the mean value of the $s / z$ score was noted, from 1.61 to 1.25 . The $t$ value is 17.53 and the $p$ value is $<0.00001$, which is statistically significant.

In subjective rating by VRQOL questionnaire, the pretreatment value was 32.85 which improved to 22 . The $t$ value is 24.02 and the $p$ value is $<0.00001$, which is statistically significant. 
Table 1: Abnormal stroboscopic parameters before and after treatment

\begin{tabular}{llclc}
\hline & Pretreatment & Posttreatment & p value & Tvalue \\
\hline Glottic closure & 85 & 17 & $<0.00001$ & -40.12 \\
Mucosal wave & 32 & 7 & $<0.00001$ & -5.91 \\
Symmetry & 44 & 12 & $<0.00001$ & -24.01 \\
Periodicity & 53 & 9 & $<0.00001$ & -17.09 \\
Amplitude & 46 & 8 & $<0.00001$ & -14.81 \\
\hline
\end{tabular}

\section{Video-stroboscopic Assessment}

There was an observable improvement in all video-stroboscopic parameters-glottis closure, mucosal wave amplitude, periodicity, and symmetry (Table 1). All the patients showed incomplete glottic closure $(n=85)$, with an hourglass pattern found most commonly $(n=56)$, which improved after treatment in 68 cases and sustained in 17 cases. A focal absence of mucosal wave was observed in 32 patients, which reduced to 7 cases after treatment. The mucosal wave asymmetry was observed in 44 cases which reduced to 12 cases after treatment. The periodicity of the mucosal wave was deviated from normal in 53 cases and this was sustained in 9 cases after treatment. The amplitude of mucosal wave was observed to be deviated from normal in 46 cases, which remained in 8 cases after treatment.

\section{Discussion}

In the present study, we observed a male preponderance (62.3\%) with a male to female ratio of 1.65 . Hegde et al. ${ }^{12}$ and Shrestha et al. $^{13}$ also observed a male predominance with M:F ratio of 3:1 and $2.5: 1$, respectively.

We observed most of the cases belonged to the age group between 31 years and 40 years (31.5\%). Similar age distribution was seen in the study of Hegde et al. ${ }^{12}$ and by Hassoun et al. ${ }^{14}$

In our study, 62 out of 85 cases accepted having a history of vocal abuse. Nerurkar et al. also observed that majority of cases had history of vocal abuse. ${ }^{15}$

In the present study, 42 cases (49.4\%) were smokers. Hassoun et al. ${ }^{14}$ encountered a history of smoking in $25 \%$ of cases while Guha et al. reported a positive history of smoking in $54 \%$ of cases. ${ }^{16}$

The most common lesions observed in the present study are vocal fold nodules. Hardianingwati and Ardani ${ }^{17}$ also reported vocal nodules to be the most commonly observed lesions (65\%) cases.

However, Hegde et al. ${ }^{12}$ noted vocal polyps to be the most commonly found lesion.

In the present study, the most common site of lesion was anterior one-third ( $n=54)$. Diffuse involvement of vocal folds was observed in 15 cases, middle third in 13 cases, and posterior third was affected only in 3 cases. Hardianingwati and Ardani also observed lesions most commonly in anterior one-third (85\% cases), middle one-third (15\% cases). ${ }^{17}$

\section{Video-stroboscopic Analysis}

We observed statistically significant improvement in all stroboscopic parameters after treatment. Thomas et al. ${ }^{2}$ studied 30 cases and Hassoun et al. ${ }^{14}$ studied 18 cases and noted statistically significant improvement in various stroboscopic parameters after treatment.

All the patients showed incomplete glottic closure $(n=85)$, with an hourglass pattern found most commonly $(n=56)$, which improved after treatment in 68 cases and sustained in 17 cases. Thomas et al. ${ }^{2}$ observed all (30) cases with incomplete glottic closure before treatment and it was improved in 29 cases after treatment. Hassoun et al. ${ }^{14}$ observed significant improvement in the duration of glottis closure after treatment.

We noted the focal absence of mucosal wave in 32 patients, which reduced to 7 cases after treatment. Similar to our observation, Thomas et al. ${ }^{2}$ found significant improvement in the mucosal wave.

The mucosal wave asymmetry was observed in 44 cases which reduced to 12 cases after treatment. The results of Thomas et al. ${ }^{2}$ and Hassoun et al., ${ }^{14}$ in agreement with our study, showed statistically significant improvement in mucosal wave symmetry.

The periodicity of the mucosal wave was deviated from normal in 53 cases and this was sustained in 9 cases after treatment. In accordance with our study, Hassoun et al. ${ }^{14}$ also noted significant improvement in the mucosal wave.

Similarly, in our study, the amplitude of mucosal wave improved in 38 and persisted in 8 cases after treatment. Hassoun et al. ${ }^{14}$ observed improvement in 16 out of 18 cases after treatment.

\section{Voice Analysis}

In our study, MPT improved from a mean of 8.2 seconds to a mean of 17.5 seconds after treatment. The $t$ value is 34.65 and the $p$ value is $<0.00001$, which is statistically significant. Singh et al. ${ }^{18}$ observed improvement in MPT from 8.58 to 12.46 seconds at 3 months posttreatment.

Prasad et al. noted an improvement in MPT from $8.73 \pm 1.72$ seconds to $10.88 \pm 1.80,11.60 \pm 1.96$, and $13.85 \pm 2.70$ posttreatment at 3,6 , and 12 weeks, respectively. ${ }^{19}$

Virmani et al. observed enhancement of MPT from 9.43 to 14.16 seconds at 3 months and 14.46 seconds at 6 months. ${ }^{20}$

We noted a significant improvement in the mean value of the $s / z$ score, from the mean value of 1.61-1.25, with a $p$ value $<0.00001$, which is statistically significant. Similar to our observation, Virmani et al. also noted a statistically significant reduction in $s / z$ from 1.37 to 1.16 at 3 months and 1.15 at 6 months. ${ }^{20}$

\section{ConClusion}

This study shows that video-stroboscopy is a reliable method of early diagnosis, identification of benign vocal fold lesions, and assessment of results of treatment. Simple methods of voice analysis aid in this assessment. As observations of both video-stroboscopy and voice assessment can be explained to the patients, it adds to their understanding of their problems and improvement over the course of treatment. This understanding also motivates them to follow up, practicing voice therapy, and suggested lifestyle modifications.

This study shows positive outcomes on all observable parameters after adequate standard treatment including coldknife microlaryngeal surgery, voice therapy, PPIs, low dose steroids, and lifestyle modifications. It can be concluded that modern treatment of such lesions starts with proper diagnosis, includes prior counseling, preoperative and postoperative voice therapy, guidance, and voice analysis at various steps till the previously set achievable goal is met. Cold-knife video-assisted microlaryngeal surgery remains a cost-effective as well as an efficient method for the treatment of vocal fold lesions. Including adequate voice therapy is important for voice improvement.

\section{Clinical Significance}

The results of this study indicate that strategy of treatment of benign vocal lesions should focus on both, the cure of lesion and acquiring a better voice quality. So, voice therapy plays an important 
role in the treatment of benign vocal lesions. Video-stroboscopy and voice analysis are helpful in diagnosis and follow-up.

\section{LiMITATIONS}

- The size of the study is small in terms of the number of cases included. A larger study may give more validity to the conclusions.

- Compliance of patients for voice therapy and suggested lifestyle modifications was variable. This had an impact on the results of the study.

\section{References}

1. Johns MM, Sataloff RT, Merati AL, et al. Shortfalls of the American Academy of Otolaryngology-Head and Neck Surgery's clinical practice guideline: hoarseness (dysphonia). Otolaryngol Head Neck Surg 2010;143(2):175-177. DOI: 10.1016/j.otohns.2010.05.026; discussion 175.

2. Thomas G, Mathews SS, Chrysolyte SB, et al. Outcome analysis of benign vocal cord lesions by videostroboscopy, acoustic analysis and voice handicap index. Indian J Otolaryngol Head Neck Surg 2007;59(4):336-340. DOI: 10.1007/s12070-007-0096-0.

3. Amitabha Roychoudhary NKN. Chapter 14: nodules and polyp. 1st ed., Jaypee Brothers Medical Publishers (P) Ltd.; 2017. pp. 147-154.

4. Stemple J. A holistic approach to voice therapy. Semin Speech Lang 2005;26(2):131-137. DOI: 10.1055/s-2005-871209.

5. Casper JK, Murry T. Voice therapy methods in dysphonia. Otolaryngol Clin North Am 2000;33(5):983-1002. DOI: 10.1016/s00306665(05)70259-0.

6. Patel RR, Romeo SD, Van Beek-King J, et al. Endoscopic evaluation of the pediatric larynx. In: McMurray JS, Hoffman MR, Braden MN, ed. Multidisciplinary management of pediatric voice and swallowing disorders [Internet]. Cham: Springer International Publishing; 2020. pp. 119-133. Available from: 10.1007/978-3-030-26191-7_14.

7. Speyer R, Bogaardt HCA, Passos VL, et al. Maximum phonation time: variability and reliability. J Voice 2010;24(3):281-284. DOI: 10.1016/j. jvoice.2008.10.004

8. Gelfer MP, Pazera JF. Maximum duration of sustained /s/ and /z/ and the $\mathrm{s} / \mathrm{z}$ ratio with controlled intensity. J Voice Off J Voice Found 2006;20(3):369-379. DOI: 10.1016/j.jvoice.2005.03.011.

9. Watkinson JC. Scott-Brown's Otorhinolaryngology Head and Neck Surgery. 8th ed., vol. 3. CRC Group; 2018. pp. 937-938.
10. Eckel FC, Boone DR. The $S / Z$ ratio as an indicator of laryngeal pathology. J Speech Hear Disord 1981;46(2):147-149. DOI: 10.1044/ jshd.4602.147.

11. Agarwal SK, Gogia S, Agarwal A, et al. Assessment of voice related quality of life and its correlation with socioeconomic status after total laryngectomy. 2015;4(4):169-175. DOI: 10.3978/j.issn.22245820.2015.09.05

12. Hegde MC, Kamath MP, Bhojwani K, et al. Benign lesions of larynx-a clinical study. Indian J Otolaryngol Head Neck Surg 2005;57(1):35. DOI: 10.1007/BF02907624.

13. Shrestha BL, Amatya RCM, Kc S, et al. Aetiological factors of hoarseness in patients attending at Kathmandu University Hospital. Bangladesh J Otorhinolaryngol 2013;19(1):14-17. DOI: 10.3329/bjo. v19i1.11877.

14. Hassoun YL, Shaheed KM, Al-Turaihy EH.Videostroboscopic evaluation of the vocal cords before and after microlaryngeal phonosurgery for benign lesions. Int J Multidiscip Curr Res 2018;6(01):Available from: http://ijmcr.com/videostroboscopic-evaluation-of-the-vocalcords-before-and-after-microlaryngeal-phonosurgery-for-benignlesions/.

15. Nerurkar NK, Chitnis T, Gupta VK, et al. A 10-year study of the etiopathogenesis of cysts with a study of seromucinous glands in vocal folds. Laryngoscope 2020;130(4):986-991. DOI: 10.1002/ lary.28206.

16. Guha R, Mondal T, Dutta M, et al. Clinico-demographic trend of benign vocal cord lesions among urban population attending tertiary care institution of Kolkata. IOSR J Dental Med Sci 2015;14(11):64-66.

17. Hardianingwati LM, Ardani DM. Prevalence of benign vocal fold lesions in ear, nose, and throat outpatient unit of Dr. Soetomo General Hospital, Surabaya, Indonesia. Biomol Health Sci J 2020;3(1):37. DOI: 10.20473/bhsj.v3i1.19103.

18. Singh A, Upadhyay A, Shrivastava A, et al. A comprehensive voice analysis in benign vocal fold lesions treated by cold knife endolaryngeal surgery using subjective, objective and videostroboscopic parameters [Internet]. Springermedizinde 2020. Available from: https://www.springermedizin.de/a-comprehensivevoice-analysis-in-benign-vocal-fold-lesions-trea/16586682.

19. Prasad S, Raychowdhury R, Roychoudhury A. Assessment of pre and postoperative voice quality in cases of vocal fold polyp. Int $J$ Otorhinolaryngol Head Neck Surg 2020;2(2):352-358. DOI: 10.18203/ issn.2454-5929.ijohns20200150.

20. Virmani N, Sharma A, Dabholkar J. Outcome analysis in patients with benign vocal fold lesions. Int J Phonosurg Laryngol 2016(1):8-13. DOI: 10.5005/jp-journals-10023-1111. 